\title{
Enhancing the autonomy of disabled persons: Assistive technologies directed by user feedback
}

\author{
N Ragot, F Bouzbouz, R Khemmar, JY Ertaud \\ ESIGELEC - IRSEEM \\ Technopôle du Madrillet, Avenue Galilée \\ 76801 Saint Etienne du Rouvray, France \\ Email: prenom.nom@esigelec.fr
}

P. Sajous, E. Niyonsaba, D. Reguer

UMR IDEES, Lab. Cirtai Université du Havre

25 rue Philippe Lebon

76600 Le Havre, France

Email: patricia.sajous@univ-lehavre.fr, niyemma75@yahoo.fr

K. Sirlantzis, G. Howells

School of Engineering and Digital Arts

University of Kent, Jennison Building

Canterbury, Kent, CT2 7NT, United Kingdom

Email: K.Sirlantzis@kent.ac.uk, W.G.J.Howells@kent.ac.uk

\author{
A-M. Kokosy \\ ISEN - LILLE \\ 41 Boulevard Vauban \\ 59800 Lille, France
}

\author{
O. Labbani-Igbida \\ UPJV, Lab. MIS \\ 33 rue Saint Leu \\ 80039 Amiens Cedex 1, France
}

Email: annemarie.kokosy@isen-lille.frEmail: ouiddad.labbani@u-picardie.fr

\begin{abstract}
Europe faces a major and growing healthcare problem due to increase in population, increasing longevity and an aging population with disability. Such dependent, elderly, disabled and vulnerable persons, are concerned since they wish to live at home as long as possible. This aspiration is also shared by national policies and communities across EU. To ensure the optimum care of dependent people, innovative solutions are encouraged to maintain independent life style. This paper outlines two projects, SYSIASS and COALAS, which aim to develop a set of technology based solutions to meet the needs and empower these people by enhancing mobility and communication.
\end{abstract}

\section{INTRODUCTION}

The European countries are facing major socio-economic challenges due to:

- Increased life expectancy and disability along with a shortage of caregivers [1], is leading to an increase in demand for social care both from family and from the welfare states of EU;

- A prevalence of chronic neuro-disability which is steadily increasing. Approximately 10 million people in UK have disabilities with a neurological diagnosis. The cost of caring for neuro-disabled persons in Europe has been estimated as 795 Billion Euro [2];

- A prevalence of wheelchair users which is increasing in Western countries, estimated in the last decade at $60-200$ per 10,000 . The numbers of such users are expected to increase with improved survival from neurological conditions e.g. $80 \%$ of people with spinal cord injury are expected to depend on a wheelchair for the rest of their lives.

\author{
H. Hu, K. McDonald-Maier \\ School of Computer Science and Electronic Engineering \\ University of Essex, Wivenhoe Park \\ Colchester, CO4 3SQ, United Kingdom \\ Email: hhu@essex.ac.uk, kdm@essex.ac.uk
}

\section{Pepper, M. Sakel}

East Kent Hospitals University NHS Foundation Trust Kent and Canterbury Hospital, Ethelbert Road Canterbury, Kent, CT1 3NG, United Kingdom

Email: matthew.pepper@nhs.net,msakel@nhs.net

Governments and healthcare services are under pressure, facing an important challenge: offering to the users solutions to their needs. Nowadays, innovative solutions emerge through the use of assistive technology which allows the user to enhance his independence, increase his access to societal role and his quality of life [3]. Within this framework, the SYSIASS and COALAS projects bring solutions to these issues by designing and developing innovative systems to assist the user in his everyday life. The projects are based on a close relation between social and technical sciences, so that the users needs are taken into account upstream of the developments. Therefore, social sciences are no longer limited only to investigations of social reception but are an integral part of the development of specifications via exploratory surveys that will identify user expectations and consider the relevance to the scale at which technologies should be implemented. This paper is organized as follows : section II gives a general overview of the projects and details the key actions. Section III presents the results obtained in terms of social surveys and technological developments. A conclusion and perspectives are given in section IV.

\section{SYSIASS AND COALAS PROJECTS}

\section{A. Project overview}

The SYSIASS project, December 2010 until June 2014, is supported by the INTERREG IVA 2 Seas programme and aims to design and implement new devices for a powered wheelchair. These devices need to be adapted, customised and personalized to the users and their carers needs. Those need to ahave the ability to interface with any commercially available powered wheelchair. They allow the users to easily 
navigate and communicate within indoor spaces (eg: home, hospital or company) and increase their autonomy. Three types of devices are proposed: navigation assistance, secure communication and multi-modal hands-free human-machine interfaces (cf. figure 1). The development of all devices is realized in collaboration with users.. Through translational clinical trials, such devices will be evaluated for efficacy performances, efficiency, usability and acceptability of new technologies. The COALAS project, November 2012 until June 2015, is supported by European cross-border programme INTERREG IVA France (Channel) England and focuses on creating an autonomous cognitive platform, combining an intelligent wheelchair coupled with the assistive capabilities of a humanoid robot (specifically NAO robots). SYSIASS and COALAS projects complement each other. In particular, the developments related to the powered wheelchair through the SYSIASS project should prefigure those made COALAS. Moreover, COALAS project should also open up new technological fields related to humanoid robotics as the cognitive platform should integrate with a robot (eg: NAO).

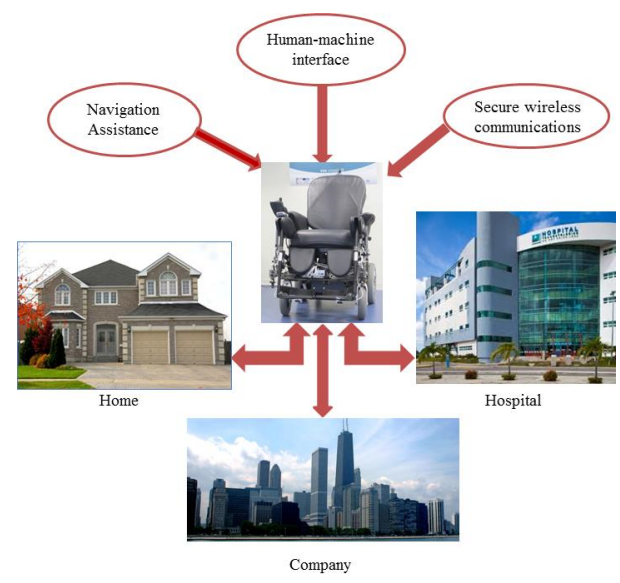

Fig. 1. Intelligent powered wheelchair proposed by SYSIASS project

\section{B. Key actions}

1) Social survey: The social survey focussed on the role of technologies in the social life of dependent people. It assessed the needs of the client and their carers. The social survey was organized around qualitative interviews (30: family, home helper, nursing auxiliary, nurse, doctor, etc.), conducted around three "situations": someone in a wheelchair (electric or manual), someone walking with difficulty using a walker or support bars, Alzheimer patient or related, requiring regular monitoring (of disease).

2) Guidance assistance device: Several intelligent wheelchairs have been proposed in the literature [4], [5]. Their development or use have not progressed due to economic reasons or because the developments do not adequatly answer the needs of the users. On the basis of these observations, the SYSIASS project proposes three different versions of guidance. The first one only guarantees collision avoidance but does not help the user to avoid obstacles by correcting the wheelchairs trajectory. In the second, the device corrects the wheelchairs trajectory if the user is not able to do so it itself. In the third version, the device ensures an autonomous navigation for the wheelchair. Through the COALAS project, vision sensors will be integrated on the wheelchair and used to ensure autonomous navigation in complex indoor and outdoor environments. They will also be used for recognition tasks by using biometrics recognition and automatic tracking of people. According to the requirements, needs and services for robotic assistive technologies, different scenarios with variable levels of autonomy are considered, in order to adapt to the physical functions and disability of the patient : obstacle avoidance, autonomous localization and navigation for target tracking and path following in crowded indoor and outdoor environments, ability to move and navigate in large environments without requiring human assistance.

3) Secure communication devices: A further major strand of the project SYSIASS and COALAS is to develop means of ensuring that secure communications can be initiated and maintained between the powered chair and other medical devices present within the system. To achieve this, the project has adapted a technology developed by the partners to derive digital signatures and encryptions keys directly from the operating characteristics of the devices themselves, a technology termed ICmetrics due to its analogy with human biometrics. ICMetrics represents a process of eliminating the need to store an encryption key on a system, by employing properties from which to generate an encryption key from the hardware characteristics of the powered chair together with the software executing upon it. Again analogously with a biometric system, a number of samples are taken from the device. The primary purpose of the research is to detect evesdropping or tapering with the system via malware, the presence of which would cause a generated digital signature to be modified.

4) HMI: The current electric powered wheelchairs (EPWs) are mostly joy stick driven and require specialised controls in order to be used by disabled and elderly people whose autonomy is seriously affected by spinal cord injuries, tetraplegia or amputation, as well as aging. Therefore, it is necessary to develop a novel human machine interface (HMI) for hands-free control of EPWs. Up to now, a number of hands-free HMIs have been developed for disabled and elderly people to control EPWs by using voice, gesture, tongue movements, eye tracking and facial expressions etc. [6]. One aim of the SYSIASS and COALAS projects will deal with the development of the hands-free control of an EPW using facial expressions which generate three control models, depending on the ability of the user in performing facial expressions (description given in section III-C): C-Mode 1 (2 facial expressions), C-Mode 2 (3 facial expressions) and C-Mode 3 (4 facial expressions). These expressions are detected by using the "cognitiv suite" of the Emotiv sensor with good accuracy. The Emotiv EPOC headset is used in the bio-signal HMI to detect facial expressions and generate three control modes. It is a device that measures EEG activity from 14 saline electrodes (Figure 2). Consequently, the "cognitive suite" is employed for training the facial expressions that would be used for the control commands.

5) Humanoid Robot: In the recent years, the humanoid robotics has emerged around the topic of disability and dependancy. Therefore, the COALAS project is part of this area of innovation by aiming to develop the assistive capabilities of a humanoid robot (NAO robot for instance). The use of these autonomous intelligent systems may reduce significantly the cost 


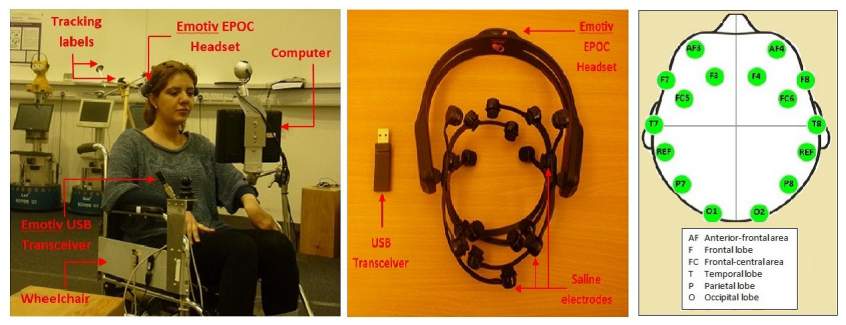

Fig. 2. Proposed hardware architecture for the intelligent powered wheelchair

of providing remote care-services by reducing or eliminating the need for human caregivers when not required by the user. This solution can provide objective monitoring capabilities and round the clock supervision, being easily scalable and having the possibility of centralisation (i.e. in-home systems with a remote central unit of supervision, such as a hospital). The system can provide the option for remote communication with health-care specialists for online consultations and diagnoses, and routine check-ups. By reducing the need to travel to specific locations, it allows users any-time contact with the care-service providers from the comfort of their own home. Also, by using a centralised communication infrastructure such systems improve the efficiency of the health-care services.

\section{RESULTS}

The results presented in section are part of the works made in the SYSIASS and COALAS projects for the social survey. Regarding the techological developments, only results obtained through the SYSIASS project are presented, as no technological implementations are done yet in COALAS.

\section{A. Social survey}

Initial interviews shed light into the social context of the users. That context determines the need, acceptability of the assistive systems (wheelchair and the humanoid robot) and improvement of which is the ultimate aim of health and social care.

- $\quad$ The people with disablility tend to have less outdoor mobility which is often the need for full participation in life roles including occupational activities and pastime which often are similar to people who live in institutions.

- Different factors of motor disabilities are mentioned: chronic diseases, accidents (falls), decrease of physical capacities. These factors could be added in some cases.

- Mobility disabilities could stem from a multitudes of impairments eg muscle weakness of upper and lower limbs, poor balance and lack of confidence due to fear of falls or wheelchair accidents.

- Two types of motor disabilities should be considered: disabilities associated with gross motor skill that prevent to move the body, and disabilities in the hands.

Secondly, interviews provide the first reactions about technical propositions of assistive systems.
- In the sample, nobody wants to substitute the care helper (family, friends and professional caregivers) by assistive systems. Sometimes, there is reluctance to accept assistive systems when it appears that they are being introduced for those reasons.

- Nevertheless, assistive systems seem like an attractive option to provide some solutions when the disabled person is alone. They can be seen not as a substitute for human help, but as a valued assistant. An intelligent system could detect some technical anomalies and correct them automatically. At home, assistive systems would bring complementary support to the helper. Thus, the helper should spend more time for dialogue and exchange with the disabled person. That means, assistive systems will not have the same knowhow as the care helper but their missions can focus on bringing various types of help when the disabled person is alone.

\section{B. Guidance assistance device}

The first prototype of the guidance assistance device has been tested by 27 users at Hospital of Garches (France) divided into 3 groups: 9 healthy volunteers who have never driven an electric wheelchair, 9 experienced electric wheelchair users and 9 users who did not pass the electric wheelchair drivers licence. They evaluated it in a created circuit (see Figure 3) which simulates a real life indoor environment (doors, walls, obstacles). The evaluation criteria were the number of collisions during the test and the time necessary for the user to finish the test. The device used information from 9 US and 2

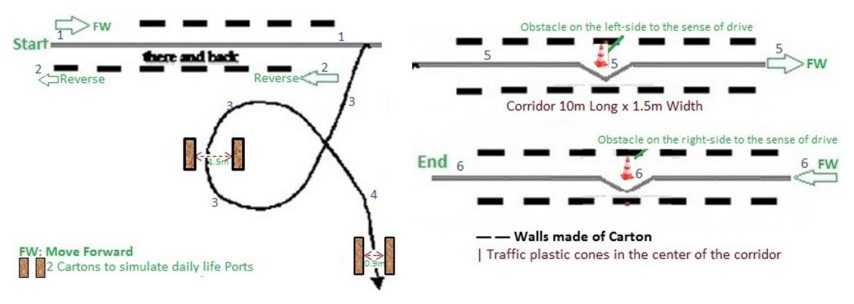

Fig. 3. Circuit of evaluation at the Hospital of Garches (France)

IR sensors to detect the presence of obstacles in the path of the wheelchair and then slowed down or stopped the wheelchair depending on the distance from the obstacle. The system implemented in an electrical wheelchair manufactured by a French company, DupontMedical. The users found this device useful for the reversing. However, they asked to have more help from the intelligent device, for example by correcting the wheelchairs trajectory when they are not able to do so themselves. So, to reach the users needs, a second version of the device is under development. This will be implemented in an electrical wheelchair manufactured by Invacare. It uses the same sensors to help the user to correct the wheelchair trajectory to avoid obstacles. This second prototype will be tested at the East Kent Hospitals at Canterbury (UK). The users who will evaluate it are assumed to be able to drive a powered chair using a joystick in most environments. Moreover, they know which room they are in, and where the doorways are. Therefore they are able to plan their path to do the door. 


\section{Human machine interface}

Two healthy subjects (a female and a male) have operated the wheelchair employing three and two different control modes, respectively. Five experiments were carried out by each control mode. In each experiment, the subject has to follow the route shown in figure 4 without hitting obstacles. All modes were tested at a slow speed of $15 \mathrm{~cm}$ per second for safety. Nine facial expressions were employed in the experiments: BEC, closing both eyes and doing one deep breath - FB, furrowing the brows without closing eyes - LEC, closing left eye only; LS, pulling the left side of the lip to the left - PJ, pulling and exerting force on the muscles of the jaw - RB, pulling the right side of the lip to the right - REC, closing right eye only - RS, pulling the right side of the lip to the right $-\mathrm{S}$, doing a big smile.

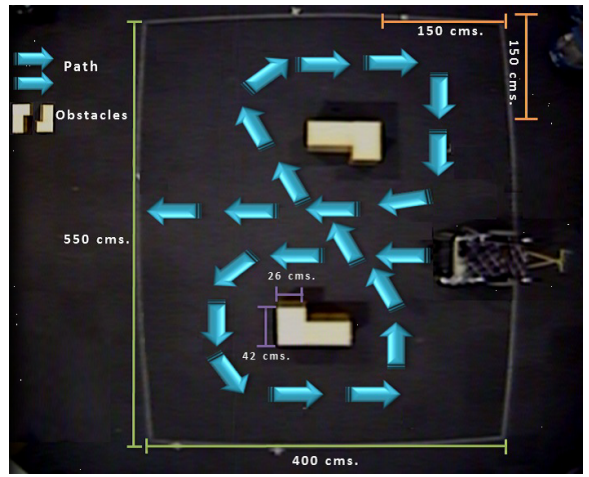

Fig. 4. The route specified for the bio-control of the wheelchair

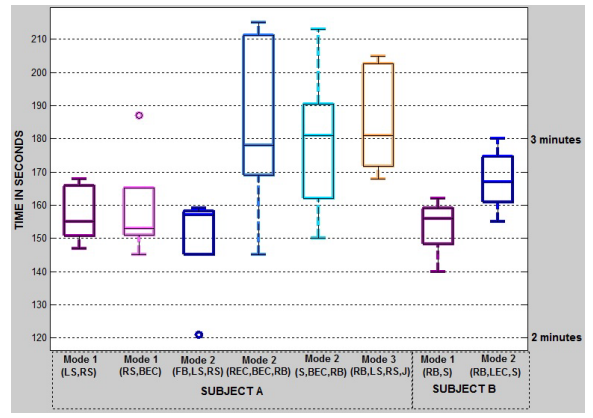

Fig. 5. Travel times of Subjects A and B at different control modes with different facial expressions

The wheelchair travel times and trajectories were recorded. Means and standard deviations of the travel times were calculated. The position of the wheelchair was obtained by a motion tracking system, VICON, which tracks the five markers attached to the wheelchair. To achieve a good performance of the control modes, the Emotiv sensor has to be attached properly to the users head, for this reason a head band was used. In all control modes the electrodes have to be deployed. Figure 5 shows the box plots of the travel times of both subjects in the different control modes using several facial expressions. Considering minimum and maximum travel times between both subjects, the smallest of the minimum times was of 121 seconds, achieved by Subject A using C-Mode 2 with $\mathrm{FB}, \mathrm{LS}$, and RS, whereas the largest of the maximum times was of 215 seconds, corresponding to Subject A employing
C-Mode 2 with REC, BEC and RB. But, if only the minimum travel times of the C-Mode 1 are considered, Subject B had the smallest one, being of 140 seconds. It is clear that the proposed bio-signal based HMI has the characteristic of being flexible, allowing the user to train and record his desired facial expressions, as well as select the number of them that wants to employ. Additionally, in all control modes the user does not have to maintain the facial expression for a long time in order to keep running the control command, therefore becoming more comfortable. After experiments, it can be concluded that the "cognitiv suite" of Emotiv EPOC is reliable for training facial expressions; moreover, it is clear that C-Mode 1 and C-Mode 2 are reliable for controlling the wheelchair using different facial expressions. The future work will be focused on the implementation of an extended hybrid HMI in which the user can choose the manner of providing the control command either using facial expressions or head movements.

\section{CONCLUSION}

This paper outlined two distinct projects, SYSIASS and COALAS, within the scope of developing innovative technologies for improving the autonomy of disable persons while taking into account the needs of the users via a social survey. The prototypes of these novel assistive devices and HMI will be evaluated through formal clinical trial in the East Kent University Foundation Hospitals Trust by June 2014 involving people with neuro-disability. Regarding the COALAS project, working groups have emerged in response to social survey, which should allow the developments of innovative technologies for the cognitive platform.

\section{ACKNOWLEDGMENT}

These research works have been implemented within the frame of the SYSIASS and COALAS projects which are part-funded by the European Union (ERDF) via the Interreg IVA 2 Seas programme and Franco-British programme. Also, the SYSIASS project would like to thank the medical staffs from Hospital of Garches and especially Bruno Guillon and Professor Frédéric Lofaso, who have been done the clinical trials and the Fondation of Garches who funded them.

\section{REFERENCES}

[1] E. Torta, J. Oberzaucher, F. Werner, R. H. Cuijpers, and J. F. Juola, "Attitudes towards socially assistive robots in intelligent homes: Results from laboratory studies and field trials," Journal of Human-Robot Interaction, vol. 1(2), pp. 76-99, 2012.

[2] A. Gustavsson, M. Svensson, and F. Jacobi, "Cost of disorders of the brain in europe 2010," European neuropsychopharmacology : the journal of the European College of Neuropsychopharmacology, vol. 21, pp. 718$779,2011$.

[3] "Medical rehabilitation in 2011 and beyond. report of a working party," Royal College of Physicians, Tech. Rep., 2011.

[4] D. Vanhooydonck, E. Demeester, A. Hntemann, J.Philips, G. Vanacker, H. V. Brussel, and M. Nuttin, "Adaptable navigational assistance for intelligent wheelchairs by means of an implicit personalized user model," Robotics and Autonomous Systems, vol. 58, pp. 963-977, 2010.

[5] M. R. M. Tomari, Y. Kobayashi, and Y. Kuno, "Development of smart wheelchair system for a user with severe motor impairment," in International Symposium on Robotics and Intelligent Sensors 2012, vol. 41, 2012, pp. 538-546.

[6] E. Rechy-Ramirez, H. Hu, and K. McDonald-Maier, "Head movements based control of an intelligent wheelchair in an indoor environment," in IEEE Int. Conf. on Robotics and Biomimetics, 2012. 